\title{
AVALIAÇÃO DA EVAPOTRANSPIRAÇÃO POTENCIAL EM ÁREA DE MATA ATLÂNTICA, PARQUE ESTADUAL DAS FONTES DO IPIRANGA, SÃO PAULO, SP
}

\author{
Frederico Luiz FUNARI
}

Augusto José PEREIRA FILHO

\begin{abstract}
RESUMO
A finalidade deste trabalho é determinar a evapotranspiração potencial em uma área do bioma de Mata Atlântica, inserida na Região Metropolitana de São Paulo (RMSP), a partir de medições obtidas em estações meteorológicas convencional e automática instaladas no Parque Estadual das Fontes do Ipiranga (PEFI), em São Paulo, SP. O cálculo da evapotranspiração potencial foi determinado a partir da partição do balanço de energia, usando o método da razão de Bowen, e pelo método Penman-Monteith, padrão recomendado pela FAO - Food and Agriculture Organization. Os valores obtidos foram 3,1 $\mathrm{mm}$ pelo balanço de energia e 3,0 $\mathrm{mm}$ pelo método Penman-Monteith, com alto coeficiente de correlação $(r=0,9467)$. Os valores são semelhantes aos obtidos em outras áreas da Mata Atlântica, como na Ilha do Cardoso (2,8 mm) e em Cunha (2,8 mm), ambos no estado de São Paulo.
\end{abstract}

Palavras-chave: Mata Atlântica; Evapotranspiração; Balanço de energia; Razão de Bowen.

\begin{abstract}
EVALUATION OF THE POTENTIAL EVAPOTRANSPIRATION IN AN ATLANTIC FOREST AREA, PARQUE ESTADUAL DAS FONTES DO IPIRANGA, SÃO PAULO, STATE OF SÃO PAULO, BRAZIL. This paper aims to determine evapotranspiration in an Atlantic Forest area located in the São Paulo Metropolitan Region, based on measurements obtained by conventional and automatic meteorological stations installed on the Fontes do Ipiranga State Park in São Paulo city in Brazil. The potential evapotranspiration was calculated based on the energy balance partition method using Bowen ratio and Penman-Monteith method, as recommended by the Food and Agriculture Organization method. The values obtained were $3.1 \mathrm{~mm}$ for energy balance and $3.0 \mathrm{~mm}$ by the Penman-Monteith method, resulting in a high correlation coefficient $(r=0.9467)$. The values were similar to those obtained in other Atlantic Forest areas, such as Ilha do Cardoso $(2.8 \mathrm{~mm})$ and Cunha $(2.8 \mathrm{~mm})$, both in the State of São Paulo.
\end{abstract}

Keywords: Atlantic Forest; Evapotranspiration; Energy balance; Bowen ratio.

\section{INTRODUÇÃO}

A transferência de moléculas de água para a atmosfera ocorre de duas maneiras: por evaporação da água em uma superfície e por transpiração das superfícies vegetadas. A perda combinada resultante desses dois processos é denominada evapotranspiração. A evaporação da água é influenciada pela temperatura, potencial de umidade, radiação solar, vento, nebulosidade, horas de brilho solar e saldo do balanço de radiação, assim como aspectos relacionados à umidade do solo e da vegetação. A evapotranspiração potencial representa, simplificadamente, a quantidade de chuva ideal para os vegetais, enquanto a evapotranspiração real é aquela que realmente ocorre em função da água disponível, e é obtida 
através do balanço hídrico, que pode ser medido ou estimado.

O conceito de evapotranspiração potencial foi introduzido por WILM et al. (1944) e THORNTHWAITE (1948) para indicar a evapotranspiração que normalmente se verifica em um terreno livremente exposto à atmosfera e inteiramente vegetado, onde não ocorra restrição hídrica para as plantas e para o solo.

A metodologia usada para a obtenção de valores de evapotranspiração pode ser realizada por medidas diretas através de evapotranspirômetros, lisímetros, tanques evaporimétricos de diversas dimensões; ou indiretamente, com o emprego de equações que utilizam dados medidos em estações meteorológicas, tais como temperatura, umidade relativa do ar, vento, radiação solar, insolação, saldo do balanço de radiação, temperatura do solo e pressão atmosférica. A equação de PenmanMonteith (ALLEN et al. 2006) (vide itens 3.2.2 e 3.2.3) é considerada padrão para o estudo da evapotranspiração pela FAO - Food and Agriculture Organization, pois considera fatores energéticos e aerodinâmicos. ALLEN et al. (2006) apresentam um coeficiente de cultura $(\mathrm{Kc})$ de cálculos padronizados para diversos cultivos agrícolas em suas várias fases fenológicas, porém não cita o Kc para a vegetação natural. $\mathrm{O}$ trabalho de MONTEIRO et al. (2016) fornece valores de Kc para várias espécies em florestas tropicais e que existem na Mata Atlântica e na nossa área de estudo.

O objetivo deste trabalho é comparar a evapotranspiração calculada pelo método padrão de Penman-Monteith (ALLEN et al. 2006) com o método do balanço de energia, usando a razão de Bowen (1926). Ressalta-se a pouca quantidade de trabalhos sobre evapotranspiração em áreas de Mata Atlântica usando esses métodos. Realizou-se também uma comparação dos resultados obtidos neste trabalho com outros obtidos em outras áreas da Mata Atlântica.

\section{2 ÁREA DE ESTUDO}

A área escolhida para esta pesquisa foi $o$ Parque Estadual das Fontes do Ipiranga (PEFI), localizado na parte sul da cidade de São Paulo, SP. O PEFI é uma reserva de Mata Atlântica inserida na RMSP (Região Metropolitana de São Paulo), em meio à densa área urbana da cidade (Figura 1). A cidade de São Paulo está inserida na província geomorfológica do Planalto Atlântico, na zona do Planalto Paulistano (ALMEIDA 2018), em sua maior parte sobre a bacia sedimentar cenozoica de São Paulo. O PEFI situa-se na transição entre a bacia sedimentar de São Paulo e os terrenos cristalinos pré-cambrianos, em altitudes que variam de 840 a $780 \mathrm{~m}$. Dista, aproximadamente, a $45 \mathrm{~km}$ do oceano Atlântico a sudeste da área.
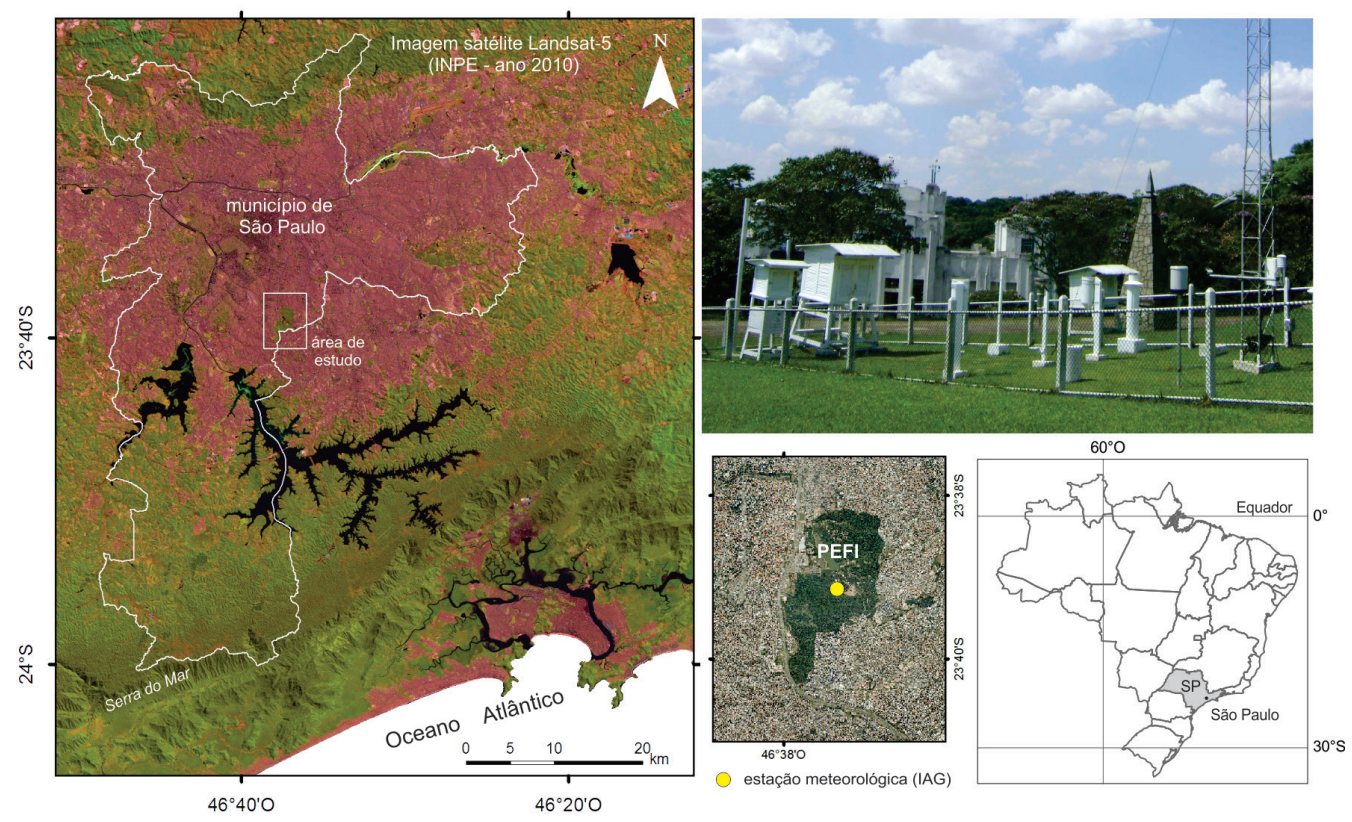

FIGURA 1 - Localização da área de estudo e detalhe do cercado meteorológico onde foi realizado o experimento. 
Essas características físicas implicam em um sítio urbano aberto e convergente, conforme proposta de classificação dos sítios urbanos elaborada por MONTEIRO (1990). No interior do PEFI - Parque Estadual das Fontes do Ipiranga, encontra-se o Parque Cientec-USP, onde se acham instaladas as Estações Meteorológicas Convencional (EMC) e Automática (EMA), pertencentes ao IAG Instituto de Astronomia, Geofísica e Ciências Atmosféricas da Universidade de São Paulo.

Por estar próximo à costa oriental do continente sulamericano e junto ao Trópico de Capricórnio, o PEFI está em uma área de transição climática, entre os climas tropicais, com período seco definido, e os subtropicais, permanentemente úmidos do Brasil (TARIFA \& ARMANI 2001). O clima da cidade de São Paulo é classificado como tropical úmido de altitude (TARIFA \& ARMANI 2001), com um regime pluviométrico de concentração de chuvas na primavera e verão, com redução no outono e inverno, apresentando um total anual pluviométrico médio de $1.402,8 \mathrm{~mm}$ (1933-2014) (IAG 2015). A pluviosidade nessa área é influenciada durante a primavera-verão pela atuação da ZCAS - Zona de Convergência do Atlântico Sul (VILELLA 2017) e pela frente polar Atlântica no outono-inverno (MONTEIRO 1973). A radiação global média anual diária (19612007) é de 187,2 W.m ${ }^{-2}$ e a insolação anual, de 1879 h.ano ${ }^{-1}\left(5.25\right.$ h.dia $\left.^{-1}\right)$. O saldo do balanço de radiação apresenta média anual diária de 114,5 W. $\mathrm{m}^{-2}$ (FUNARI \& TARIFA 2017).

O PEFI tem uma área de 526,38 ha, sendo recoberto por um importante remanescente de vegetação natural da Mata Atlântica. Na parte central do PEFI estão instaladas a Estação Meteorológica Convencional (EMC) do IAGUSP, com início das observações em 01/01/1933, e, desde 27/09/2007, a Estação Meteorológica Automática (EMA), com sensores de todos os elementos da convencional, além do sensor de saldo do balanço de radiação. Ambas estão instaladas no mesmo cercado meteorológico, situado na latitude $23^{\circ} 39^{\prime} 04^{\prime \prime} \mathrm{S}$, longitude 463' $21^{\prime}$ ' W, na altitude 802,0 m (Figura 2).

A área foi alvo de inúmeros trabalhos sobre aspectos do meio físico, incluindo estudos sobre o clima e características biológicas (MARQUES DOS SANTOS \& FUNARI 1976, 2002; BICUDO et al. 2002; PEREIRA FILHO et al. 2007; FUNARI $\&$ PEREIRA FILHO 2010, 2014, 2017).
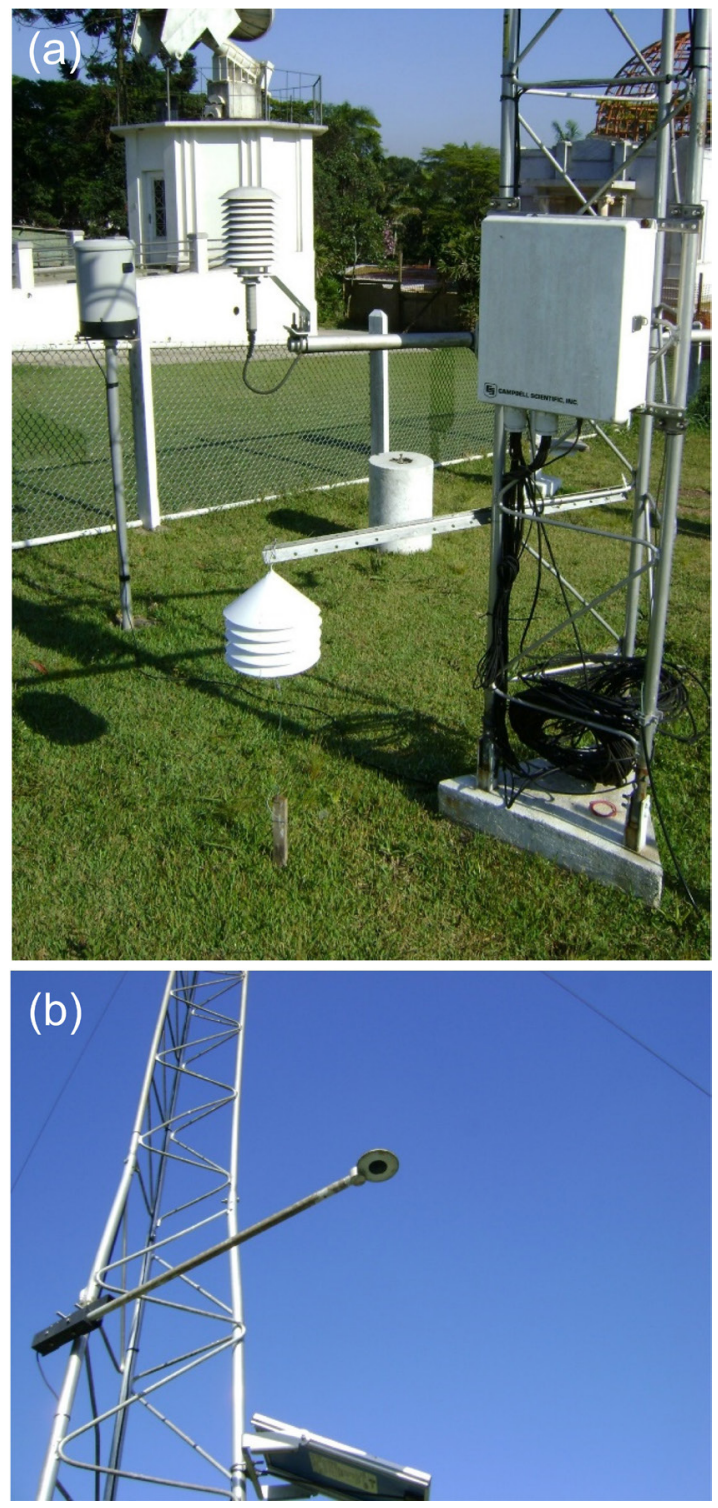

FIGURA 2 - a) Instrumentos (abrigos com termohigrômetros em duas alturas: $1,5 \mathrm{~m}$ e $0,80 \mathrm{~m}$ ). b) Saldo radiômetro para obtenção do balanço de energia.

\section{MATERIAL E MÉTODOS}

\subsection{Material}

\subsubsection{Variáveis meteorológicas}

A maior parte dos dados usados neste trabalho são provenientes da Estação EMA, mas também se utilizou dados provenientes da EMC. Para a determinação da Razão de Bowen há a necessidade de registros de temperatura do ar 
e temperatura de bulbo úmido em dois níveis de altura diferentes. Na EMA, temperatura e umidade do ar são registrados na altura padrão de $1,5 \mathrm{~m}$. Adicionalmente foi instalado na altura de 0,8 $\mathrm{m}$ outro registrador autônomo (datalogger HT500), que fornece a temperatura do ar e umidade relativa do ar. Esse registrador foi instalado em um abrigo de ventilação passiva desenvolvido por ARMANI \& GALVANI (2006). A partir dos dados de temperatura do ar e umidade relativa do ar, empregando-se a Tabela Psicrométrica de Jelinek, foram obtidas as temperaturas dos termômetros úmidos e também os valores de pressão atual e de saturação do vapor (es e ea).

$\mathrm{O}$ registro das variáveis meteorológicas na EMA ocorre a cada 5 minutos, enquanto da EMC a cada hora. Os dados da EMA foram reduzidos para horários (médias aritméticas simples) para ficarem compatíveis com a resolução dos dados da EMC. As variáveis meteorológicas empregadas no trabalho e as características dos elementos sensores são apresentadas na tabela 1 .

Este instrumental, cujo funcionamento foi estudado e comparado com a EMC, foi objeto do trabalho de FUNARI \& PEREIRA FILHO (2010), obtendo-se correlação entre as medições bastante alta: acima de 0,94 e chegando, em alguns casos, a 1,0 .
3.1.2 Série temporal e critério para seleção de dias para análise

O período estudado foi de março de 2011 a janeiro de 2017, perfazendo um total de 125 dias. Restringiu-se o horário para análise dos dados entre as $08 \mathrm{~h}$ e $16 \mathrm{~h}$ para minimizar erros provenientes dos gradientes de temperatura do ar e termômetro úmido (PEREZ et al. 1999), devido à altura do Sol, que está bastante baixa tanto no nascer como no ocaso.

Os valores de insolação relativa $(\mathrm{n} / \mathrm{N})$ devem ser preferencialmente acima de 0,50. Os valores superiores a 0,5 da razão de Bowen foram desconsiderados, porque podem indicar condição de instabilidade atmosférica - possível advecção de calor sensível para a área experimental, caso não haja bordadura suficiente para garantir que os fluxos sejam apenas verticais (GALVANI \& ESCOBEDO 2001). Outro fator determinante, que restringiu o número de dias, foi a nebulosidade elevada.

\subsection{Métodos}

Existem vários métodos para se obter a evapotranspiração. Dos métodos diretos destacamse os evapotranspirômetros, lisímetros, tanques de evaporação, sendo os mais conhecidos o Tanque Classe A (Weather Bureau) e o Tanque de $20 \mathrm{~m}^{2}$. Ainda nessa linha empírica, existem atmômetros de Pichè, Livingtone, Belani; além de evaporímetros e evaporígrafos. Os métodos indiretos são aqueles

TABELA 1 - Variáveis meteorológicas empregadas e propriedades dos sensores.

\begin{tabular}{llcccc}
\hline \multicolumn{1}{c}{ Variável } & \multicolumn{1}{c}{ Sensor } & Modelo & Fabricante & Precisão / Unidade & Estação Meteorológica \\
\hline Temperatura $(0,80 \mathrm{~m})$ & $\begin{array}{l}\text { Termistor } \\
\text { capacitiva }\end{array}$ & HT - 500 & Instrutherm & $0,1{ }^{\circ} \mathrm{C}$ & EMA \\
Temperatura do ar $(1,5 \mathrm{~m})$ & $\begin{array}{l}\text { Resistência } \\
\text { de platina }\end{array}$ & HPM 45 C & Vaisala & $0,2^{\circ} \mathrm{C}$ & EMA \\
Umidade relativa do ar $(0,80 \mathrm{~m})$ & $\begin{array}{l}\text { Termistor } \\
\text { capacitiva }\end{array}$ & HT - 500 & Instrutherm & $3 \%$ & EMA \\
Umidade relativa do ar $(1,5 \mathrm{~m})$ & Capacitiva & HPM $45 \mathrm{C}$ & Vaisala & $5 \%$ & EMA \\
Temperatura do solo & Termistor & 107 & Campbell & $0,5{ }^{\circ} \mathrm{C}$ & EMA \\
Pressão atmosférica & Silicon cap. & CS 105 & Vaisala & $2 \mathrm{hPa}$ & EMC \\
Radiação solar & Bimetálico & $58 \mathrm{c}$ & R.Fuess & $1,5 \%$ & EMC \\
Saldo do balanço de radiação & Termopilha & NR-light-NET & Campbell & $<30 \mathrm{~W} \cdot \mathrm{m}^{-2}$ & EMA \\
Insolação & Heliógrafo & Campbell-Stokes & W.Lambrecht & $0,1 \mathrm{~h}$ & EMC \\
Vento & $\begin{array}{l}\text { Direção } \\
\text { Velocidade }\end{array}$ & $05103-5$ & Young MI & $2,0^{\circ} / 1 \mathrm{~m} \cdot \mathrm{s}^{-1}$ & EMA \\
Chuva & Báscula & TB 4 & Campbell & $0,2 \mathrm{~mm}$ & EMA \\
\hline
\end{tabular}


que se utilizam de medições meteorológicas de variáveis como temperatura, umidade relativa, vento, radiação solar, saldo do balanço de radiação, insolação, em equações diversas, com a finalidade de obter a evapotranspiração.

Apesar da existência de diversos métodos, foram escolhidos o método do Balanço de Energia, também designado como razão de Bowen (1926), e o método padrão Penman-Monteith (ALLEN et al. 2006), em função de serem os mais abrangentes, pois incluem a parte energética e aerodinâmica da evapotranspiração.

3.2.1 Método do Balanço de Energia ou de Razão de Bowen

O saldo do balanço de radiação pode ser descrito como a somatória dos fluxos de calor sensível, latente de evaporação, no solo, para realização da fotossíntese e para respiração vegetal, aquecimentos das plantas e outros processos, conforme equação 1 :

$$
R n=H+L E+G+F+M(\text { Eq. 1) }
$$

Sendo:

$R n$, o saldo do balanço de radiação $\left(\mathrm{W} \cdot \mathrm{m}^{-2}\right)$;

$H$, o fluxo de calor sensível para aquecimento do ar (W. $\left.\mathrm{m}^{-2}\right)$; $\left(\mathrm{W} \cdot \mathrm{m}^{-2}\right)$;

$L E$, o fluxo de calor latente de evaporação

$G$, o fluxo de calor no solo (W.m ${ }^{-2}$ );

$F$, o fluxo de calor para realização da fotossíntese $\left(\mathrm{W} . \mathrm{m}^{-2}\right)$;

$M$, o fluxo de calor para respiração vegetal, aquecimento das plantas e outros processos (W.m. ${ }^{-2}$.

Os valores percentuais de $F$ e $M$ são baixos, da ordem de $1 \%$ de $R n$. $G$ é também baixo, representando cerca de $3 \%$ de $R n$ (FUNARI \& PEREIRA FILHO 2017). Portanto os processos mais significativos são $H$ e $L E(95 \%) . L E$ geralmente é maior em áreas vegetadas, e representa frequentemente mais da metade do total de $R n$.

As leis que regem o fluxo de calor nos gases não podem ser aplicadas para predizer o fluxo de calor sensível na atmosfera, pois é um sistema aberto. O fluxo de ar acima da superfície do solo faz com ele seja continuamente renovado e misturado com o ar das camadas mais altas. A técnica experimental tem dificuldades em solucionar este problema porque a medida de $H$ é muito difícil. Para resolver este impasse usam-se artifícios como a Razão de BOWEN (1926), que também permite calcular o valor de $L E$.

BOWEN (1926) definiu a relação entre os fluxos de calor sensível $(H)$ e calor latente $(L E)$ para calcular os fluxos turbulentos de calor sensível e calor latente em uma superfície natural. Para tanto é necessário que se conheça o saldo do balanço de radiação na superfície $(R n)$, o fluxo de calor no solo $(G)$, a diferença das leituras de termômetros úmidos $\left(\Delta T U=T U_{2}-T U_{1}\right)$ e secos $\left(\Delta T=\Delta T_{2}-T_{1}\right)$ colocados em 2 níveis altimétricos, a temperatura média úmida $(M T U)$ e o valor médio de $\left(T U_{2}+\right.$ $\left.T U_{1}\right)$. Este método também é designado como Razão de Bowen $(B)$.

Conforme demonstrado na Eq. 1 podemos assumir que na prática o saldo do balanço de radiação corresponde:

$$
R n=H+L E \text { (Eq. 2) }
$$

A Razão de BOWEN (1926) é equacionada na sua forma mais simples:

$$
B=H / L E \text { (Eq. 3) }
$$

O valor de $B$, já reduzido à sua forma simplificada por VILLA NOVA (1973), pode ser descrito conforme a equação 4 :

$$
B=1 /[(s+\gamma / \gamma) *(\Delta T U / \Delta T)]^{-1}(\text { Eq. } 4)
$$

sendo:

$s$, tangente à curva de saturação de vapor no ponto da temperatura média úmida (MTU), ou $\left(\mathrm{TU}_{2}+\mathrm{TU}_{1}\right) / 2$;

$\gamma$, constante psicrométrica $\left(0,47 \mathrm{mmHg} .{ }^{\circ} \mathrm{C}^{-1}\right)$ ou $0,6266 \mathrm{hPa}$;

$(s+\gamma) / \gamma)$, provenientes de tabelas já calculadas por VILLA NOVA (1973) e FERREIRA (1972);

$\Delta T U\left(T U_{2}-T U_{1}\right)$, a diferença de temperatura de bulbo úmido medidas nos níveis $Z_{2}$ e $Z_{1}$;

$\Delta T\left(T_{2}-T_{1}\right)$, a diferença das temperaturas seca medidas nos níveis $Z_{2}$ e $Z_{l}$;

$Z_{1}$ e $Z_{2}$, correspondem aos níveis de $0,80 \mathrm{~m}$ e $1,5 \mathrm{~m}$, respectivamente.

A equação 5 permite calcular o fluxo de calor latente $(L E)$ sobre uma superfície natural, desde que se conheça $R n, G, \Delta T U$ e $\Delta T$ e os níveis $Z_{2}$ e $Z_{1}$.

$$
L E=(R n+G) / 1+B(\text { Eq. 5) }
$$

O valor de $G$ é geralmente obtido por sensores que medem o fluxo de calor no solo usando termopilhas (fluxímetros). No presente estudo, o 
cálculo de $G$ foi realizado conforme descrito em FUNARI \& PEREIRA FILHO (2017).

\subsubsection{Método de Penman-Monteith (ALLEN} et al. 2006), PADRÃO - FAO

O método de PENMAN (1956) foi aperfeiçoado por MONTEITH (1965), que complementou com parâmetros de fisiologia vegetal. A equação original de PENMAN (1956) para a evaporação potencial é descrita como:

$$
E P=[(\Delta / \Delta+\gamma) * R n / 59]+[(\gamma / \Delta+\gamma) * E a](\text { Eq. 6) }
$$

Sendo:

$\Delta$, tangente à curva de saturação do vapor; $\gamma$, constante psicrométrica;

$R n$, saldo do balanço de radiação (W.m $\mathrm{m}^{-2}$ );

$E a$, termo aerodiânico da formulação, obtido pela equação 7 :

$$
E a=0,35 *(e s-e a) *\left(0,5+0,54 U_{2}\right)(\text { Eq. } 7)
$$

Sendo:

(es-ea), o déficit de saturação;

$e s$, a pressão de saturação do vapor (hPa);

$e a$, a pressão atual do vapor $(\mathrm{hPa})$;

$U_{2}$, a velocidade do vento $\mathrm{m} \cdot \mathrm{s}^{-1}$ a $2 \mathrm{~m}$.

A equação Penman-Monteith considera a parte energética e a fisiologia vegetal para a determinação da evapotranspiração para as culturas agrícolas, por esse motivo ela é considerada "padrão" pela FAO.

Assim, a equação para cálculo da Evapotranspiração de Referência pelo Método de Penman-Monteith - FAO é descrita pela equação 8:

$$
E T o=\frac{0,408 * \Delta(R n-G)+\gamma(900 / T+273) U_{2}(e s-e a)}{\Delta+\gamma\left(1+0,34 U_{2}\right)}(\text { Eq. 8) }
$$

Sendo que:

$E T o$, é a evapotranspiração de referência segundo Penman-Montheith (mm.dia $\left.{ }^{-1}\right)$

$\Delta$, é o declive da curva de saturação do vapor $\left(\mathrm{kPa} .{ }^{\circ} \mathrm{C}^{-1}\right)$;

$R n$, o saldo do balanço de radiação à superfície de referência $\left(\mathrm{W} \cdot \mathrm{m}^{-2}\right)$;

$G$, o fluxo de calor no solo (W.m $\left.{ }^{-2}\right)$;

$\gamma$, a constante psicrométrica $\left(0,06168 \mathrm{kPa} .{ }^{\circ} \mathrm{C}^{-1}\right)$;

$900, K c$ o coeficiente para a cultura de referência de cálculos padronizados;

$T$, a temperatura média do ar $\left({ }^{\circ} \mathrm{C}\right)$;

$U_{2}$, a velocidade média do vento a uma altura de $2 \mathrm{~m}\left(\mathrm{~m} \cdot \mathrm{s}^{-1}\right)$; $(e s-e a)$ o déficit de pressão do vapor $(\mathrm{kPa})$; 0,408 o valor para $1 / \lambda$, assumindo $\lambda$ equivalente a 2,45 MJ.kg-1;

0,34 o coeficiente de vento para a cultura de referência $\left(\mathrm{kJ}^{\mathrm{kg}} \mathrm{kg}^{-1} \cdot \mathrm{K}^{-1}\right)$.

\subsubsection{Determinação do Coeficiente de Cultu-} ra $(\mathrm{Kc})$

O valor obtido pela equação de PenmanMonteith (ALLEN et al. 2006) corresponde a um local padronizado com grama. Portanto, para outros tipos de coberturas do solo é necessária a aplicação de um valor de correção, chamado genericamente de coeficiente de cultura $(K c)$. Não existem valores específicos para áreas com florestas, sendo necessário pesquisar valores de coeficiente de cultura $(K c)$ para várias espécies existentes na floresta que se pretende pesquisar. No caso do PEFI, com sua cobertura do solo de Mata Atlântica, levantou-se valores de $K c$ das principais espécies de árvores existentes no parque, conforme tabela 2, baseada em MONTEIRO et al. (2016). Apesar dos resultados desse estudo serem para mudas na transição Cerrado-Amazônia, ela foi a única referência encontrada sobre $K c$ dessas espécies. Tendo em vista que essas espécies são frequentes no PEFI, adotou-se como valor de $K c$ para o PEFI a média aritmética de todos os valores da tabela $2(0,69)$ para ajuste dos valores de $E P$ pelo método de Penman-Monteith.

TABELA 2 - Valores de Kc e de EP para as principais espécies que existem na área do PEFI, conforme MONTEIRO et al. (2016).

\begin{tabular}{llcc}
\hline Nome popular & Nome cientifico & Kc & $\begin{array}{c}E P \\
\left(\mathrm{~mm} \mathrm{dia}^{-1}\right)\end{array}$ \\
\hline Pau d'Arco & Tabebuia Impetiginosa & 0,75 & 2,90 \\
Ipê Branco & Tabebuia Rosealba & 0,63 & 2,46 \\
Ipê Amarelo & Handroanthus Chrysotrichus & 0,74 & 2,84 \\
Olho de Pavão & Adenanthera Pavonina & 0,65 & 2,53 \\
Média & & 0,69 & 2,68 \\
\hline
\end{tabular}

Assim, para corrigir os valores de evapotranspiração deste trabalho (área de Floresta/ Mata Atlântica) aplicou-se a equação 9:

$E P=E T o * K c$ (Eq. 9)

Sendo:

$E P$, a evapotranspiração da vegetação no PEFI (mm.dia $\left.{ }^{-1}\right)$;

ETo, a evapotranspiração segundo a Equação Padrão (FAO) (mm.dia $\left.{ }^{-1}\right)$; 
Também foram calculados os valores de EP para o PEFI com e sem a correção do Kc para ilustrar a necessidade de correção dos dados.

\section{RESULTADOS E DISCUSSÃO}

Os resultados do balanço de energia no período estudado (março de 2011 a janeiro de 2017) são apresentados na tabela 3 .

$\mathrm{Na}$ tabela 4 e figura 3 é possível comparar os valores de evapotranspiração potencial entre os métodos de balanço de energia e o de Penman-Montheith. Os valores são bastante próximos e apresentam elevado coeficiente de correlação $(\mathrm{r}=$ $0,9467)$, conforme pode ser visualizado na figura 4 .

Primeiramente, é necessário considerar o reduzido número de dias, principalmente em função

TABELA 3 - Resultados do balanço de energia no período de março de 2011 a janeiro de 2017 (dados registrados das $08 \mathrm{~h}$ às $16 \mathrm{~h}$ (devido à pouca altura do Sol). Qg = Radiação solar global ao nível do solo $\left(\mathrm{W} \cdot \mathrm{m}^{-2}\right), \mathrm{Rn}=$ Saldo do balanço de radiação $\left(\mathrm{W} \cdot \mathrm{m}^{-2}\right)$, $\mathrm{n} / \mathrm{N}=$ Razão de insolação (insolação medida / insolação teórica), $\mathrm{LE}=$ Calor latente de evaporação (W.m$\left.{ }^{2}\right)$ e em relação ao saldo de radiação ( $\left.\mathrm{LE} / \mathrm{Rn}-\%\right)$, $\mathrm{EVP}=$ valor da evapotranspiração $(\mathrm{mm} / \mathrm{dia}), \mathrm{G}=$ Fluxo de calor no solo (W.m ${ }^{-2}$ ) e em relação ao saldo de radiação $(\mathrm{G} / \mathrm{Rn}-\%), \mathrm{H}=$ fluxo de calor sensível $\left(W^{-2}\right), H=(R n-L E-G), B=$ valor da razão de Bowen $(B=H / L E)$.

\begin{tabular}{lrrrr}
\hline Variáveis & Outono & Inverno & Primavera & Verão \\
\hline $\begin{array}{l}\text { Número de } \\
\text { dias utilizados }\end{array}$ & 29 & 28 & 29 & 39 \\
Qg $\left(\mathrm{W} \cdot \mathrm{m}^{-2}\right)$ & 203,6 & 169,9 & 251,8 & 283,9 \\
Rn $\left(\mathrm{W} \cdot \mathrm{m}^{-2}\right)$ & 110,4 & 79,6 & 147,5 & 167,9 \\
n/N & 0,67 & 0,76 & 0,63 & 0,65 \\
LE (W.m $\left.{ }^{-2}\right)$ & 74,4 & 52,6 & 104,2 & 121,5 \\
LE (\%) & 67,4 & 66,0 & 70,7 & 72,4 \\
EVP (mm) & 2,6 & 1,8 & 3,6 & 4,2 \\
G (W.m $\left.{ }^{-2}\right)$ & 3,4 & 4,3 & 2,7 & 1,7 \\
G (\%) & 3,1 & 5,4 & 1,8 & 1,0 \\
H (W.m $\left.{ }^{-2}\right)$ & 32,6 & 22,8 & 40,5 & 44,7 \\
H (\%) & 29,5 & 28,6 & 27,5 & 26,6 \\
B & 0,43 & 0,42 & 0,39 & 0,36 \\
\hline
\end{tabular}

de vários apresentarem a razão de Bowen acima de 0,5 . Outro fator decisivo é a frequência elevada de nebulosidade, o que ocasionou dias com pouco saldo do balanço de radiação e baixa altura do Sol, o que restringiu o horário de estudo diário das $8 \mathrm{~h}$ às $16 \mathrm{~h}$, hora local.

A análise de regressão linear demonstrou alta correlação e determinação entre os dois métodos empregados. Nesse trabalho também foi possível constatar a qualidade dos dados coletados oriundos da EMA, principalmente dos sensores, tal como observado por FUNARI \& PEREIRA FILHO (2010). O datalogger HT-500 da Instrutherm instalado em abrigo de ventilação passiva (ARMANI \& GALVANI 2006) mostrou-se também de qualidade muito satisfatória e dentro das faixas de erro do HT $500\left(0,2{ }^{\circ} \mathrm{C}\right.$ para temperatura, $3 \%$ para Umidade relativa do ar).

Comparando-se com outras áreas de Mata Atlântica no Estado de São Paulo (Tabela 5), observou-se que os resultados são semelhantes e da mesma ordem de grandeza, a despeito da utilização de métodos diversos, inclusive com as medições de evapotranspiração diretas através de evapotranspirômetros tipo Thornthwaite (CAMARGO 1966).

TABELA 4 - Comparação dos valores médios de Evapotranspiração Potencial calculados a partir dos métodos Balanço de Energia e o método padrão da FAO (ALLEN et al. 2006). Valores em mm $\left(\mathrm{H}_{2} \mathrm{O}\right)$.

\begin{tabular}{lcc}
\hline & Balanço de energia & Penman-Monteith (*) \\
\hline Outono (29 dias) & 2,6 & 2,5 \\
Inverno (28 dias) & 1,8 & 1,9 \\
Primavera (29 dias) & 3,6 & 3,7 \\
Verão (39 dias) & 4,2 & 4,0 \\
Ano (125 dias) & 3,1 & 3,0 \\
\hline (*) valores corrigidos para o coeficiente de cultura da Mata \\
Atlântica (Kc=0,69). \\
\multicolumn{3}{c}{ 5 CONCLUSÕES }
\end{tabular}

A partir dos resultados obtidos no presente trabalho pode-se concluir que:

- A metodologia escolhida para a avaliação da evapotranspiração potencial apresentou resultados satisfatórios, comprovado pelos elevados índices de correlação entre os métodos do balanço de energia, obtido a partir Razão de BOWEN (1926) quando comparada com o método Padrão da FAO 

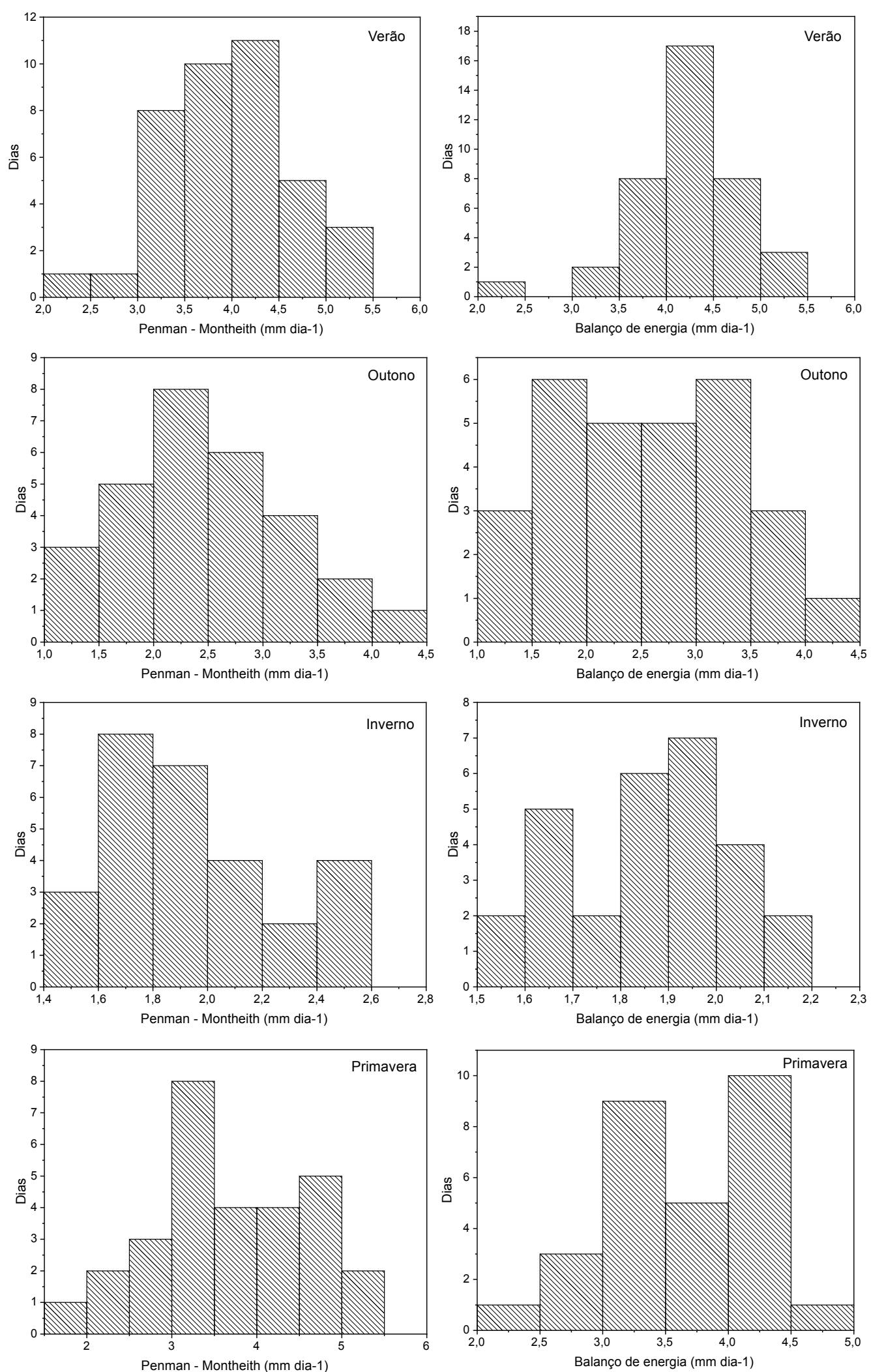

FIGURA 3 - Histogramas de evapotranspiração potencial calculados pelos métodos de Penman-Montheith e de balanço de energia. 

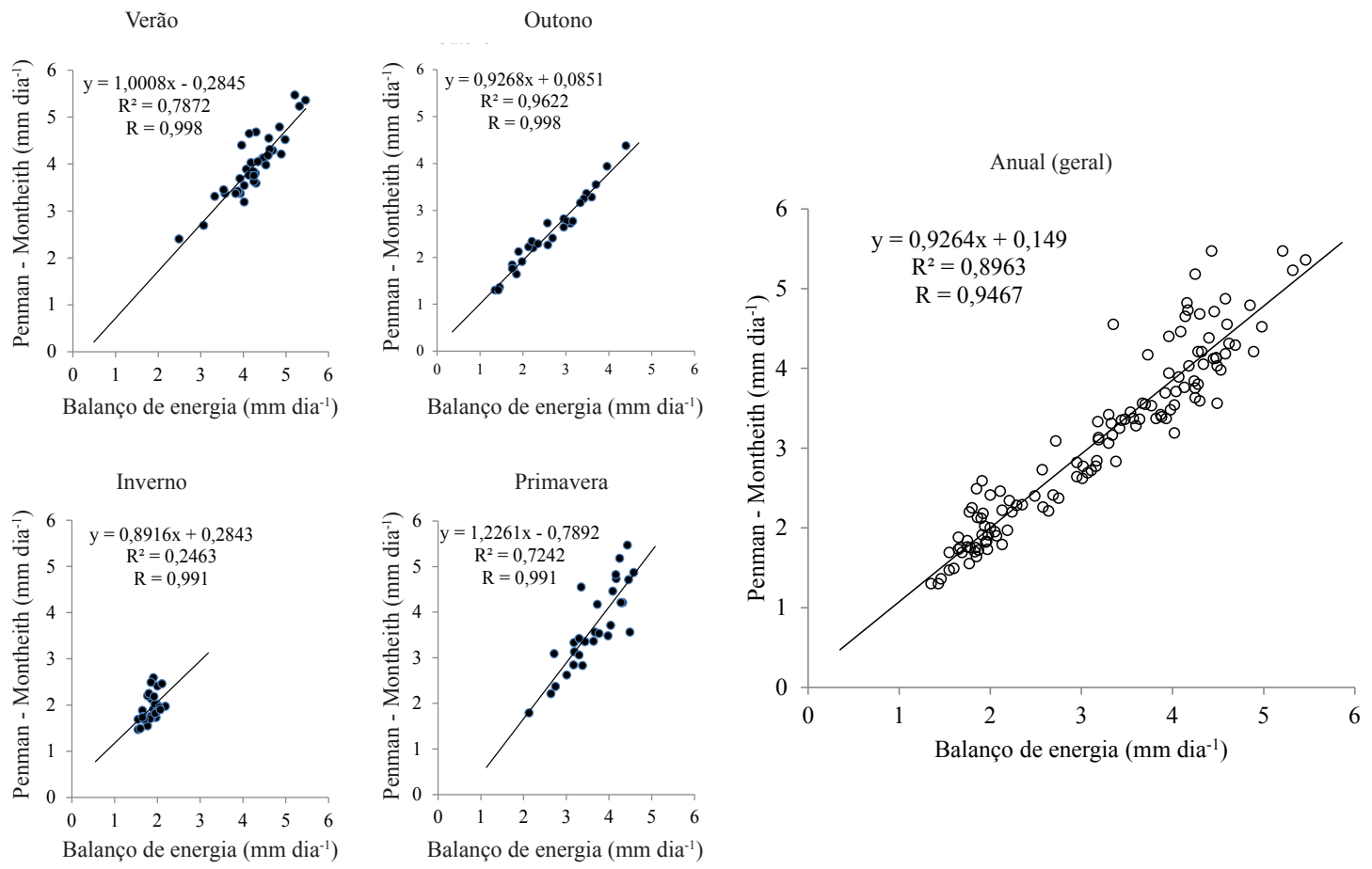

FIGURA 4 - Correlação entre os valores de evapotranspiração potencial calculados pelo Balanço de Energia (abscissa) e Penman-Monteith (ordenada) em mm dia ${ }^{-1}$. Gráficos elaborados para cada estação do ano e geral (Número de pontos $=125$, Equação de regressão anual: $\mathrm{PM}=(0,9264 * \mathrm{BE})+0,149\left(\mathrm{R}^{2}=0,8963\right)$.

TABELA 5 - Medidas de evapotranspiração em áreas de Mata Atlântica do Estado de São Paulo.

\begin{tabular}{llllc}
\hline Local & Período & Método & Valor (mm/dia) & Referência \\
\hline São Paulo & $1957 / 1966$ & Thornthwaite & 2,3 & 1 \\
São Paulo & $1933 / 1972$ & Thornthwaite & 2,2 & 2 \\
Mogi-Guaçu-SP & $1971 / 1980$ & Thornthwaite & 2,7 & 3 \\
Paranapiacaba-SP & $1945 / 1968$ & Thornthwaite \& Mather & 2,3 & 4 \\
Ilha do Cardoso-SP & $1956 / 1975$ & Thornthwaite \& Mather & 2,8 & 5 \\
São Paulo-SP & $1933 / 1999$ & Thornthwaite & 2,4 & 6 \\
São Paulo-SP & $1999 / 2000$ & Balanço hídrico (Vazão) & 2,6 & 7 \\
São Paulo-SP & $2011 / 2013$ & Balanço de energia & 2,3 & 8 \\
Cunha-SP & $1983-1998$ & Thornthwaite \& Mather & 2,8 & 9 \\
Serra da Mantiqueira-MG & fev.-ago. 2008 & Penman-Monteith & 3,9 & 10 \\
& & & 3,4 Pindamonhangaba (SP) (1954/1957) & 11 \\
Estado de São Paulo & $1954 / 1959$ & Evapotranspirômetros & 3,3 Ribeirão Preto (SP) (1955/1959) & 11 \\
& & & 2,6 Campinas (SP) (1956/1959) & 11 \\
São Paulo - SP & $2011 / 2017$ & Balanço de energia & 3,1 & 12 \\
São Paulo - SP & Idem & Penman-Monteith sem corr. por Kc 4,4 & 3,0 & 12 \\
São Paulo - SP & Idem & Penman-Monteith corr. por Kc & 3,0 &
\end{tabular}

1 - MARQUES DOS SANTOS (1968-inédito), 2 - MARQUES DOS SANTOS \& FUNARI (1976), 3 - STRUFFALDI-DE-VUONO et. al. (1986), 4 FUNARI et al. (1986), 5 - FUNARI et al. (1986), 6 - MARQUES DOS SANTOS \& FUNARI (2002), 7 - PEREIRA FILHO et al. (2002), 8 - FUNARI \& PEREIRA FILHO (2014), 9 - CICCO et al. (2007), 10 - PEREIRA et al. (2010), 11 - CAMARGO (1966), 12 -Este trabalho. 
- (ALLEN et al. 2006). A utilização do método do balanço de energia foi facilitado pela existência de medições do saldo do balanço de radiação;

- Os valores de evapotranspiração obtidos pelo balanço de energia são provenientes de medições com a utilização da Razão de Bowen, que quando comparadas com a equação padrão de Penman-Monteith (ALLEN et al. 2006), requer o emprego do fator Kc (coeficiente de cultura) para a vegetação natural do PEFI;

- Valores de evapotranspiração potencial são similares aos observados em outras áreas de Mata Atlântica.

\section{AGRADECIMENTOS}

O primeiro autor agradece a FUNDESPA Fundação de Estudos e Pesquisas Aquáticas, pelo auxílio financeiro parcial desta pesquisa através de Bolsa de Pós-Doutorado. Aos relatores da Revista do Instituto Geológico, pelas sugestões apresentadas que enriqueceram o artigo.

\section{REFERÊNCIAS BIBLIOGRÁFICAS}

ALLEN， R.G.; PEREIRA， L.S.; RAES， D.; SMITH, M. 2006. FAO Irrigation and Drainage Paper n. 56 - Crop Evapotranspiration. FAO, $300 \mathrm{p}$.

ALMEIDA, F.F.M. 2018 [1964]. Fundamentos geológicos do relevo paulista. Revista do Instituto Geológico, 39(3): 9-75. Republicado de Boletim do Instituto Geográfico e Geológico, número 41 (p. 169-263), 1964. https://doi. org/10.33958/revig.v39i3.600

ARMANI, G.; GALVANI, E. 2006. Avaliação do desempenho de um abrigo meteorológico de baixo custo. Revista Brasileira de Agrometeorologia, 14(1): 34-40.

BICUDO, D.C; FORTI, M.C.; BICUDO, C.E.M. 2002. Parque Estadual das Fontes do Ipiranga - unidade de conservação que resiste a urbanização de São Paulo. Secretaria do Meio Ambiente do Estado de São Paulo, São Paulo, $351 \mathrm{p}$.

BOWEN, I.S. 1926. The ratio of heat losses by evaporation from any water surface. Physical Review, 27: 779-787. https://doi.org/10.1103/ PhysRev.27.779
CAMARGO, A.P. 1966. Contribuição para a determinação da evapotranspiração potencial no Estado de São Paulo. Campinas: Instituto Agronômico, 56 p. (Boletim, 161).

CAMARGO, A.P. 1978. Balanço Hídrico no Estado de São Paulo. Campinas: Instituto Agronômico do Estado de São Paulo, 28 p. (Boletim, 116).

CICCO, V.; ARCOVA, F.C.S.; SHIMOMICHI, P.Y. 1989. Estimativa da evapotranspiração em bacia hidrográfica com floresta natural secundaria de Mata Atlântica. Revista do Instituto Florestal, 1(2):43-54.

CICCO, V.; FURIAN, M.F.; GUANDIQUE, M.E.G.; ARCOVA, F.C.S. \& RANZINI, M. 2007. Estimativas da evapotranspiração por floresta de Mata Atlântica em uma microbacia experimental na região de Cunha, SP. In: SIMPÓSIO BRASILEIRO DE GEOGRAFIA FÍSICA APLICADA, 12, 2007, Natal, Resumos, p. 1542-1558. CD-ROM.

FERREIRA, W.A. 1972. Utilização da evaporação Piche na estimativa da evapotranspiração potencial. UNESP, Botucatu, Tese de Doutoramento, $66 \mathrm{p}$.

FUNARI, F.L.; PEREIRA FILHO, A.J. 2010. Análise comparativa de medições de variáveis meteorológicas por estações meteorológica convencional e automática no Parque Estadual das Fontes do Ipiranga - SãoPaulo - SP. Revista Brasileira de Climatologia, 6(7): 159-167. http://dx.doi.org/10.5380/abclima. v7i0.25659

FUNARI, F.L.; PEREIRA FILHO, A.J. 2014. Energy Balance in a Patch of Atlantic Forest in São Paulo City, Brazil. JWARP, 6: 805-812. https://doi.org/10.4236/jwarp.2014.69076

FUNARI, F.L.; PEREIRA FILHO, A.J. 2017. Estimativa do fluxo de calor no solo a partir da temperatura do solo em São Paulo, SP. Revista do Instituto Geológico, 38(1): 49-57. http:// dx.doi.org/10.5935/0100-929X.20170004

FUNARI，F.L.; TARIFA，J.R. 2017. Sunshine, global radiation and net radiation in Brazil. Revista do Instituto Geológico, 38(2): 49-83. http://dx.doi.org/10.5935/0100-929X.20170004 
FUNARI, F.L.; STRUFFALDI-DE-VUONO, Y.; SALUM, S.T. 1986 Balanço Hídrico de duas áreas de Mata Atlântica: Reserva Biológica de Paranapiacaba e Parque Estadual da Ilha do Cardoso (ESP). In: CONGRESSO DA SOCIEDADE BOTÂNICA DE SÃO PAULO, 6, Anais, Campinas, p. 95-101.

GALVANI, E.; ESCOBEDO, J.F. 2001. Balanço de energia na cultura de pepineiro em ambiente natural e protegido. Bragantia, 60(2): 127-137. https://doi.org/10.1590/S000687052001000200009 .

IAG/USP - INSTITUTO ASTRONÔMICO E GEOFÍSICO/UNIVERSIDADE DE SÃO PAULO. 2015. Boletim climatológico anual da Estação Meteorológica do IAG/USP. Seção Técnica de Serviços Meteorológicos, IAG/ USP, v. 18.

MARQUES DOS SANTOS, P. 1968. Evaporação Potencial e Balanço Hídrico no Parque do Estado. Trabalho para Curso de Hidrologia EP-USP.

MARQUES DOS SANTOS, P.; FUNARI, F.L. 1976 Evapotranspiração Potencial e Balanço Hídrico no Parque do Estado, São Paulo - SP. Ciência e Cultura, 25: 1325-1329.

MARQUES DOS SANTOS, P.; FUNARI, F.L. 2002. Clima. In:D.C. Bicudo; M.C. Forti \& C.E.M. Bicudo (org.) O Parque Estadual das Fontes do Ipiranga (PEFI), capítulo 2. São Paulo, Secretaria Estadual do Meio Ambiente do Estado de São Paulo, p. 29-48.

MONTEIRO, C.A.F. 1973. A dinâmica climática e as chuvas nos estado de São Paulo: estudo geográfico sob a forma de atlas. São Paulo, IGEOG-USP, 169 p.

MONTEIRO, C.A.F. 1990. A cidade como processo derivador ambiental e a geração de um clima urbano: estratégias na abordagem geográfica. Geosul, 5(9): 80-114.

MONTEIRO, E.B.; SILVA, A.C.; SOUZA, A.P.; TANAKA, A.A.; FERNEDA, B.G.; MARTIM, C.C. 2016. Water requirements and crop coefficients of tropical forest seedlings in different shading conditions. Revista Brasileira de Engenharia Agrícola e Ambiental, 20(8):
709-715. http://dx.doi.org/10.1590/18071929/agriambi.v20n8p709-715

MONTEITH, J.L 1965. Evaporation and environment. Symposia of the Society for Experimental Biology, 19: 205-234.

PENMAN, H.L. 1956. Evaporation: an introductory survey. Netherlands Journal of Agricultural Science, 4: 9-29.

PEREIRA FILHO, A.J.; MASSAMBANI, O.; LOBO, G.A.; CASTILHANO, L.L. 2002. Balanço hídrico da Bacia Hidrográfica do IAG. In: D.C. Bicudo; M.C. Forti \& C.E.M. Bicudo (org.) O Parque Estadual das Fontes do Ipiranga (PEFI). São Paulo, Secretaria Estadual do Meio Ambiente do Estado de São Paulo, p. 201-212.

PEREIRA FILHO, A.J,; MARQUES DOS SANTOS, P.M.; XAVIER,T.M.B. 2007. Evolução do Tempo e do Clima na Região Metropolitana de São Paulo. IAG-USP, 281p.

PEREIRA, D.R.; MELLO, C.R.; SILVA, A.M.; YANAGI, S.N.M. 2010. Evapotranspiration of aerodynamic and stomatal conductance in a fragment of Atlantic Forest in Mantiqueira Range region. CERNE, 16(1): 32-40. https:// doi.org/10.1590/S0104-77602010000100004

PEREZ， P.J.; CASTELLVI, F.; IBAÑEZ, M.; ROSELL, J.I. 1999. Assessment of realiability of Bowen ratio method for partitioning fluxes. Agricultural and Forest Meteorology, 97(3): 141-150. https://doi. org/10.1016/S0168-1923(99)00080-5

STANHILL, G. 1962. The use of Piche evaporimeter in the calculation of evaporation. Quarterly Journal of the Royal Meteorological Society, 88(375): 80-82.

STRUFFALDI-DE VUONO, Y.; BATISTA, E.A.; FUNARI, F.L. 1986. Balanço Hídrico da Reserva Biológica de Mogi-Guaçu-SP. Hoehnea, 13: 73-85.

TARIFA, J.R.; ARMANI, G. 2001. Os climas 'naturais'. In: J.R. Tarifa \& T.R. Azevedo (Orgs.) Os climas na cidade de São Pauo: teoria e prática. São Paulo, GEOUSP, p.34-46 (Coleção Novos Caminhos, 4). 
THORNTHWAITE, C.W. 1948. An approach toward a rational classification of climate. Geographical Review, 38(1): 55-94. https:// doi.org/10.2307/210739

VILELLA, R.J. 2017 The South Atlantic Convergence Zone: a critical view and overview. Revista do Instituto Geológico, 38(2): 1-19. http://dx.doi.org/10.5935/0100$-929 X .20170006$

VILLA NOVA, N.A. 1973. Estudos sobre o balanço de energia em cultura de arroz.
ESALQ-USP, Piracicaba, Tese de Livre-Docência, 89 p.

WILM, H.G.; THORNTHWAITE, C.W.; COLMAN, E.A.; CUMMINGS, N.W.; CROFT, A.R.; GISBORNE, H.T.; HARDING, S.T.; HENDRICKSON, A.H.; HOOVER, M.D.; HOUK, I.E.; KITTREDGE, J.; LEE, C.H.; ROSSBY, C.-G.; SAVILLE, T.; TAYLOR, C.A. 1944. Report of the Committee on Transpiration and Evaporation, 1943-44. Transactions, American Geophysical Union, 25(5): 683-693. https://doi.org/10.1029/ TR025i005p00683

Endereço dos autores:

Frederico Luiz Funari e Augusto José Pereira Filho - Laboratório de Hidrometeorologia, Instituto de Astronomia, Geofísica e Ciências Atmosféricas, Universidade de São Paulo, Avenida Miguel Stéfano, 4200, Água Funda, CEP 04301-904, São Paulo, SP, Brasil. E-mails: funari.fred@gmail.com, augusto. pereira@iag.usp.br

Artigo submetido em 2 de março de 2020, aceito em 30 de outubro de 2020. 